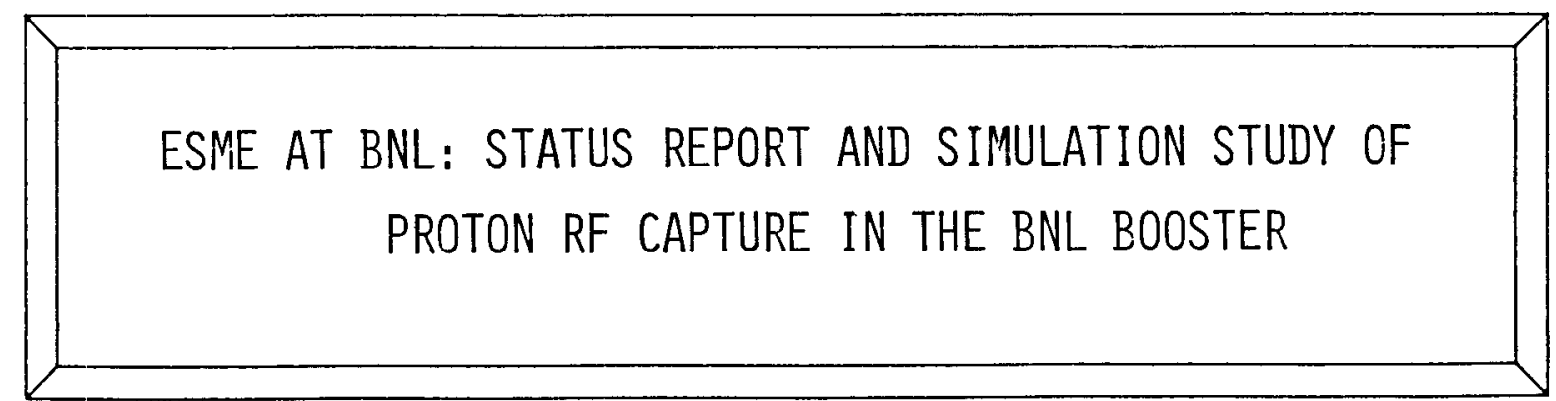

$$
\begin{gathered}
\text { AD } \\
\text { Booster Technical Note } \\
\text { No. } 118
\end{gathered}
$$

F.Z, KHIARI, A.U, LUCCIO, W.T, WENG

$$
\text { APRIL 25, } 1988
$$

ACCELERATOR DEVELOPMENT DEPARTMENT

Brookhaven National Laboratory

$$
\text { Upton, N.Y. } 11973
$$




\title{
ESME AT BNL: STATUS REPORT AND SIMULATION STUDY OF PROTON RF CAPTURE IN THE BNL BOOSTER
}

\author{
F.Z. KHIARI, A.U. LUCCIO, W.T. WENG
}

\section{Introduction}

The BNL Booster is a fast cycling accelerator designed to inject high intensity beams of protons and heavy ions into the AGS. In order to minimize the losses of these high intensity beams due to space charge effects, it is important to maximize the percentage of particles captured inside the rf bucket. Numerical simulation is the most reliable way to study this process. To this end, the computer code ESME ${ }^{[1]}$ from FERMILAB was adapted and modified over the past several months to be used at BNL as a design tool. In particular, it was used to study the physics of the capture process in the BNL Booster and its dependence on machine parameters.

In this note, we give a brief description of the main equations governing particle motion used in ESME and a brief account of the major revisions that were made to improve the FERMILAB version of ESME. We then present preliminary results of our simulation studies for the proton rf capture in the BNL Booster and, finally, conclude by mentioning some of the additional revisions we plan to incorporate in the next series of studies.

\section{ESME: Physics of Space Charge Effects}

In ESME, a number of macroparticles are tracked in the longitudinal phase space. A pair of hamiltonian difference equations is solved for each macroparticle on each turn. The percentage of particles captured inside the rf bucket is calculated as a function of the specified accelcrating voltage $V(t)$ and the time rate of change of the magnetic guide field $\dot{B}(t)$. The phase space coordinates used in ESME are the total energy $E(\mathrm{MeV})$ and the azimuth $\theta(-\pi \leq \theta \leq \pi)$ measured with respect to the energy $E$, and the azimuth $\theta_{*}$ of 
the synchronous particle. The equations of motion for particle $i$ on turn number $n$ are:

$$
\begin{gathered}
E_{i, n}=E_{i, n-1}+e V_{n} \sin \left(h \theta_{i, n}+\psi_{n}\right)+\delta E_{i, n}^{s c}, \\
\theta_{i, n}=\theta_{i, n-1}+2 \pi\left(\frac{\omega_{s}}{\omega_{i, n}}-1\right),
\end{gathered}
$$

where $\mathrm{e}$ is the proton's charge, $\mathrm{V}$ is the rf voltage amplitude, $h \equiv \omega_{r f} / \omega_{\mathrm{s}}$ is the rf harmonic number, $\psi$ is a reference phase (usually $\psi=h \theta_{s}$ ), $\omega$ is the angular velocity, and $\delta E^{s c}$ is the energy change due to space charge. This energy change is calculated for the case of a uniform cylindrical beam of radius a centered in a round pipe of radius $b$. There are two ways to calculate the space charge induced voltage. One way is to use the formula giving the space charge voltage in terms of the gradient of the linear charge density ${ }^{[2]}(\lambda(\theta))$, i.e. $\partial \lambda / \partial \theta$. The other way (used in ESME) is to use the relation $I_{b}=\beta c \lambda$ between the beam current $\left(I_{b}\right)$ and the linear charge density and $\partial I_{b} / \partial \theta=-(R / \beta c) \dot{I}_{b}$ to calculate this voltage in terms of the time derivative of the beam current $\dot{I}_{b}(t)$. R. is the radius of the equilibrium orbit of the accelerator. In addition to space charge effects, ESME can also simulate the effects of beam-wall coupling through the accelerator ring and rf impedances (these effects will be presented in a future report). The easiest way to combine these two effects is to express them both in terms of the beam current, in which case this current is Fourier analyzed and the voltage due to each beam component calculated separately. In the linear regime, the constant of proportionality between the beam current and the space charge voltage represents the complex impedance with frequency dependence. For the cylindrical configuration, the longitudinal space charge impedance ${ }^{[3]}$ is

$$
\frac{Z_{L}^{a c}}{n}=-j \frac{Z_{0} g}{2 \beta \gamma^{2}}
$$

where $n \equiv \omega / \omega_{r f}$ is the beam harmonic number, $Z_{0}=377 \Omega$ is the vacuum impedance, $\beta$ and $\gamma$ are the usual relativistic factors, and $g$ is a form factor given by ${ }^{[4]}$

$$
g=1+2 \ln \frac{b}{a}
$$


For the BNL Booster $a \approx 2.5 \mathrm{~cm}$ and $Z_{L}^{s c} / n \approx-j 700 \Omega$ at injection energy (200 MeV kinetic energy) and $Z_{L}^{* c} / n \approx-j 100 \Omega$ at extraction energy (1500 MeV kinetic energy). These are large impedances and are likely to be the main cause of beam loss in the ring. The total space charge voltage is then

$$
V^{s c}=\sum_{n} Z_{L}^{s c} I_{n}
$$

where $I_{n}$ is the $\mathrm{n}^{\text {th }}$ Fourier component of the beam current

$$
I_{n}=e N \omega_{r f} a_{n} e^{i\left(n \theta+\theta_{n}\right)}
$$

$N$ is the number of particles per bunch and $a_{n}$ and $\theta_{n}$ are the real amplitude and phase of the $\mathrm{n}^{\text {th }}$ component of the Fourier spectrum of the beam current. The energy contribution due to space charge is then

$$
\delta E^{s c}=\Re e\left(e V^{s c}\right)=e N \omega_{r f} \frac{Z_{0} g}{2 \beta \gamma^{2}} \sum_{n} n a_{n} \cos \left(n \theta+\theta_{n}-\frac{\pi}{2}\right) .
$$

For the numerical simulation, one generates the Fourier decomposition of the beam current by first binning the longitudinal distribution of the particles. The Fourier series is then obtained by a fast Fourier transform (FFT) ${ }^{[5]}$ of the bin occupation numbers. In ESME, $\delta E^{s c}$ is evaluated once per bin on each turn, i.e. $\delta E^{s c}$ is the same for all particles in a given bin. In our simulations we found some dependence of the capture efficiency on the number of bins for a given number of macroparticles. The choice of the number of bins is dictated by both physical and computational considerations. Obviously, too few bins give a poor representation of the particles' distribution. On the other hand too many bins give a good representation only if there are enough macroparticles. Moreover, the microwave cutoff consideration leads to an upper limit on the number of beam harmonics to be included in equation (7). The cutoff for the lowest $\left(T E_{11}\right)$ mode of a circular pipe of radius $b$ is ${ }^{[0]}$

$$
\lambda_{c}=3.4126 b \text {. }
$$


This wavelength $\lambda_{c}$ is Lorentz contracted by a factor $\gamma^{-1}$ when transformed to the beam frame where the fundamental interval for the Fourier decomposition is $\lambda_{r f}$, one wavelength of the accelerator rf. Therefore, the maximum number of Fourier components is

$$
n_{c}^{\max }=\frac{\lambda_{r f}}{\lambda_{c}}=\frac{\gamma \lambda_{r f}}{3.4126 b}
$$

Similarly, the maximum number of bins that is consistent with the microwave cutoff is

$$
n_{b i n}^{\max }=2 n_{c}^{\max }=\frac{2 \gamma \lambda_{r f}}{3.4126 b}
$$

This maximum number of bins corresponds to a minimum bin length, or equivalently a minimum interaction length between adjacent bins, below which the fields generated by one bin travel freely along the beam pipe at a speed different from that of the bunch and therefore do not contribute to space charge effects. For the BNL Booster $b \approx 7.5 \mathrm{~cm}$, $\gamma \approx 1.2$, and $\lambda_{r f} \approx 120 m$ for the early part of the cycle. Thus

$$
n_{b i n}^{\max } \approx 1000
$$

This number is rather large in our application, so the limit on the number of bins is generally dictated by the number of particles in the simulation and ultimately by the computing time. So we track what we could reasonably afford, $410^{4}$ particles in 128 bins in which case a typical run with space charge would take 10 to 12 hours on a VAX-8600 or a VAX-7800 with an FPS364 Array Processor.

\section{ESME: Revisions and Study of Proton rf Capture in the BNL Booster}

In addition to the extensive and time consuming work done to make ESME run in the BNL computer environment, several additions have been made to improve the FERMILAB version. We will only mention a few of the changes we have made.

The first one was to add a feature in ESME to read the voltage and magnetic field vs. time from externally supplied tables. This allows us to use any shape of of voltage and magnetic guide field. The second change in ESME was to make time the independent 
variable instead of turn number. This is more convenient since the accelerator parameters are more readily defined as a function of time. The third and more important change was to add a feature in the program that simulates multiturn injection instead of the single turn injection previously used in ESME. This is important because, in the presence of space charge, these two modes of injection yield different capture efficiencies as will be shown in the remainder of this section.

Three cases of proton of capture were studied in the BNL Booster. Multiturn and single turn injection schemes were simulated and compared both with and without space charge. The cases were classified according to their if voltage programs. Fig. 1 shows plots of the rf voltage and Fig. 2 shows plots of the $\dot{B}(t)$ curves during the first $2.5 \mathrm{~ms}$ for three cases $\mathrm{A}, \mathrm{B}$, and $\mathrm{C}$. Cases $\mathrm{A}$ and $\mathrm{C}$ were done with $\dot{B}(t)=0$ and case $\mathrm{B}$ was done with a linear $\dot{B}(t)$. For each case there were two sets of runs. One set was for multiturn injection and one set was for single turn injection. Moreover, there were two runs for each set, one run without space charge and one run with space charge ( $510^{12}$ particles/bunch). In the case of multiturn injection, the injection time was $100 \mu s,(\approx 84$ turns $)$ and the number of macroparticles as well as the number of particles per bunch were incremented each turn until they reached their final values of 40000 and $510^{12}$ respectively at $100 \mu s$ after which they were kept constant. In the absence of space charge, the number of macroparticles used was 10000 (running time $\approx 2$ hours). In all cases the injected beam from the Linac was approximated by a line beam with a kinetic energy of $200 \mathrm{MeV}$. Table 1 lists some of the other parameters used in the simulation.

Of special interest is the calculation of the capture efficiency, defined as the fraction of particles inside the rf bucket at a given time. This capture efficiency changes as the bucket area changes. Table 2 lists the capture efficiency (in \%) for the various cases. Notice that in the case of zero intensity beam ( no space charge), the capture with multiturn injection is basically the same as the capture with single turn injection. However, when space charge effects are taken into account, the difference in capture efficiency between 
the two injection schemes is significant. This difference can be attributed to the fact that space charge is incrementally built up for multiturn injection. Its effect is small at the beginning and the total effect is felt only after $100 \mu s$. In the case of single turn injection, however, the total effect of space charge is felt at the beginning. This effect is particularly strong after a quarter period of synchrotron oscillations when the longitudinal distribution of the particles in the bunch is very peaked around the synchronous particle. Fig. 3 shows plots of the bucket areas and Figs. 4.A, 4.B, and 4.C show plots of the capture efficiencies for cases $A, B$, and $C$.

Since the Linac delivers $100 \mu s$ long pulses, and because of the significant difference in capture efficiencies between multiturn and single turn injection schemes with space charge (see Table 2 and Figs. 4.A-4.C), it is necessary to use multiturn injection to realistically simulate the if capture process of high intensity beams in the Booster. To further understand the beam behavior during multiturn injection, we present the results of 3 additional simulations of proton rf capture in the Booster. The three cases $(1,2$, and 3) were done with multiturn injection only and for the voltage program shown in Fig. 5. Fig. 6 shows the $\dot{B}$ curves for these simulations. Case 1 was done with no space charge and $\dot{B}=0$. Case 2 was done with space charge ( $510^{12}$ particles/bunch) and $\dot{B}=0$. Case 3 was done with space charge and a linearly increasing $\dot{B}$ with an average of $1.5 T / s$ over the first $2 \mathrm{~ms}$. Table 3 lists the bucket areas and capture efficiencies at $2 \mathrm{~ms}$ for the 3 cases. Fig. 7 and Fig. 8 are plots of the bucket areas and capture efficiencies respectively. Figs. 9.1, 9.2 and 9.3 show the phase space plots and their $\theta$ projections at $100 \mu s$ (end of injection) and $2 \mathrm{~ms}$ for cases 1,2 , and 3 respectively. For these last three simulations, the Linac beam pulses had a uniform angular distribution and a gaussian energy distribution with $\sigma \approx 0.2 \mathrm{MeV}$.

We conclude from this study that multiturn capture is more efficient than single turn capture and that an rf capture of approximately $90 \%$ can be achieved. However, for better capture efficiency, the Linac pulses have to be chopped to eliminate those particles that 
have rf phase angles close to 0 and $2 \pi$. Work is under way to study this case.

\section{ESME: Future Revisions and Applications}

Some of the future improvements to ESME that we plan to make include:

1) Distinction between synchronous and equilibrium particles. In the present version of ESME they coincide. However, when the frequency and radius of the beam are controlled by feedback loops as planned for the Booster, the synchronous and equilibrium particles will in general differ.

2) Study of space charge effects in an elliptical geometry.

3) Elimination of the fast varying Fourier components of the beam spectrum. This amounts to making the linear charge density smoother and thereby reducing the sensitivity of the capture efficiency to the number of bins.

4) Completion of the transfer of the VAX version of ESME to the IBM 3090. This will reduce the computer running time by a factor of $\approx 5-7$.

5) Study of the capture process including the wall impedance.

Work is also in progress to find the high order modes of the Booster proton rf cavity. Once this is done, it will be possible to use ESME to study the longitudinal coupled-bunch instabilities in the Booster.

\section{Acknowledgments}

We would like to thank J. A. Maclachlan, S. Stahl and P. Lucas for providing us with the Fermilab version(s) of ESME. 


\section{REFERENCES}

[1] J. A. MacLachan, Particle Tracking in E- $\phi$ Space as a Design Tool for Cyclic Accelerators, Proceedings of The IEEE Particle Accelerator Conference, 1087 (1987)

[2] J. Wei, S. Y. Lee, A. G. Ruggeiro, RF Capture of the AGS Booster, AD Booster Technical Note No. 115 (1988)

[8] P. Lucas and J. MacLachlan, Simulation of Space Charge Effects and Transition Crossing in the Fermilab Booster, Proceedings of the IEEE Particle Accelerator Conference, 1114 (1987)

[4] The arguments for a circular chamber are also applicable to a rectangular beam pipe by using an effective $b / a$ ratio giving

$$
g_{r e c}=1+2 \ln \left(\frac{2 H}{\pi a}\right)
$$

where $H$ is the height of the rectangular pipe.

See C. E. Nielsen and A. M. Sessler, Longitudinal Space Charge Effects in Particle Accelerators, RSI, $\underline{30}, 80$ (1959)

[ъ] W. H. Press et al., Numerical Recipes, 390 (1987)

[ब] J. D. Jackson, Classical Electrodynamics, Second Edition, 356 (1975) 
Injection Energy

Linac Pulse Length

Number of Particles/Bunch

Number of Macroparticles

Number of bins

Beam Radius $a$

Beam Pipe Radius $b$

RF IIarmonic Number $h$
$200 \mathrm{MeV}$

$100 \mu s e c$

$510^{12}$

40000 (with space charge)

10000 (without space charge)

128

$2.5 \mathrm{~cm}$

$7.5 \mathrm{~cm}$

3

Table 1 Parameter List 


$\begin{array}{lllll}\text { Case } & \dot{B} & V & \text { Bucket Area } & \text { Capture } \\ & {[T / s]} & {[k V]} & {[e V . s]} & {[\%]} \\ \text { A No SC }^{\dagger}, \mathrm{MT} & 0 & 90 & 1.7 & 94 \\ \text { A No SC, ST }^{*} & 0 & 90 & 1.7 & 95 \\ \text { A SC, MT } & 0 & 90 & 1.7 & 87 \\ \text { A SC, ST } & 0 & 90 & 1.7 & 72 \\ & & & & \\ \text { B No SC, MT } & \text { Linear } & 1-90 & 1.6 & 92 \\ \text { B SC, MT } & \text { Linear } & 1-90 & 1.6 & 85 \\ \text { B SC, ST } & \text { Linear } & 1-90 & 1.6 & 83 \\ & & & & 97 \\ \text { C No SC, MT } & 0 & 45-90 & 1.7 & 98 \\ \text { C No SC, ST } & 0 & 45-90 & 1.7 & 92 \\ \text { C SC, MT } & 0 & 45-90 & 1.7 & 87 \\ \text { C SC, ST } & 0 & 45-90 & 1.7 & \end{array}$

Table 2 Listing of Capture and Bucket Area at $\approx 2.5 \mathrm{~ms}$ for Cases $\mathrm{A}, \mathrm{B}$, and $\mathrm{C}$

$\dagger \equiv$ Space Charge $\left(510^{12}\right.$ particles/bunch $)$

- $\equiv$ Multiturn Injection (100 $\mu s$ injection time)

* $\equiv$ Single Turn Injection 


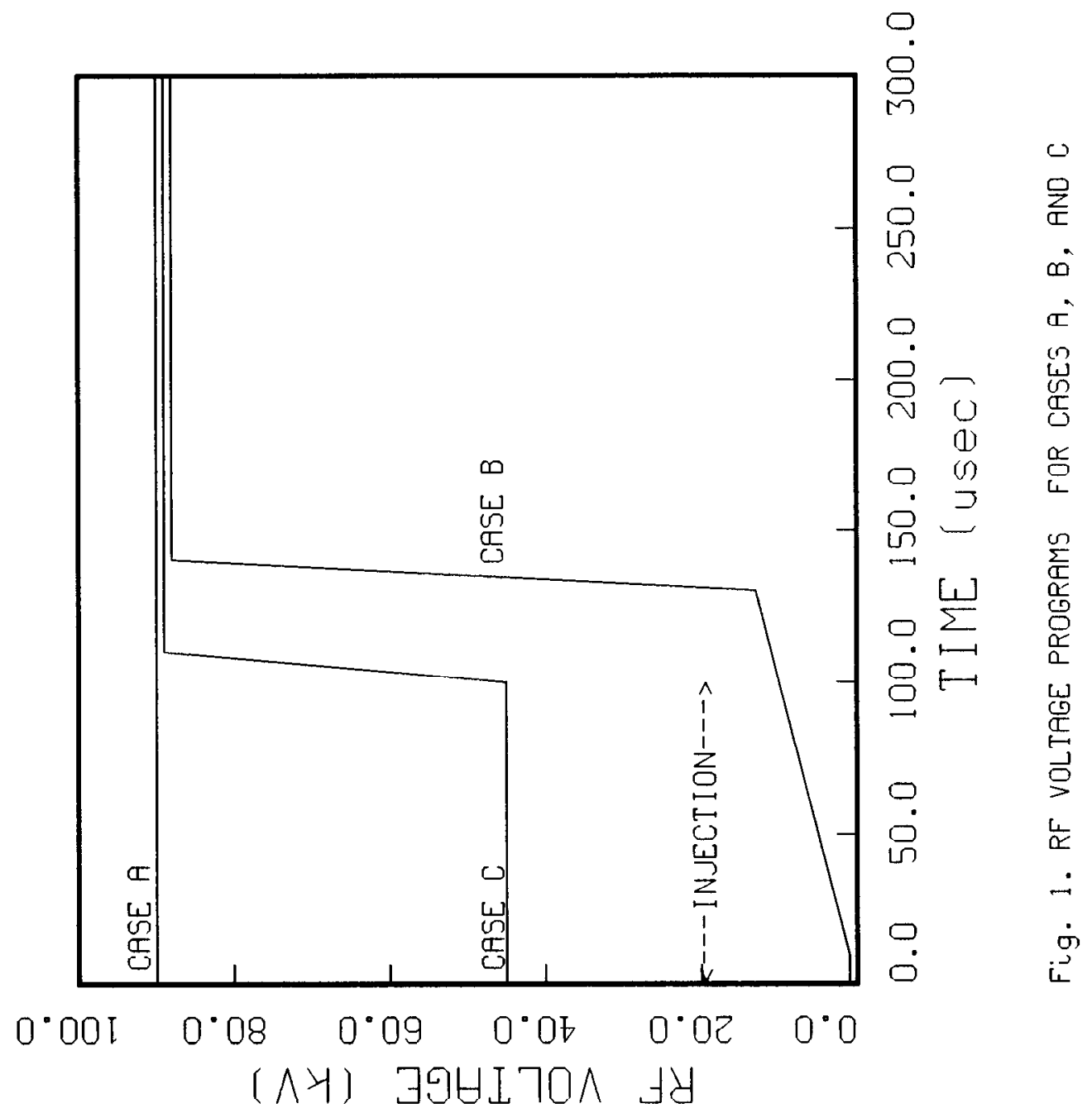




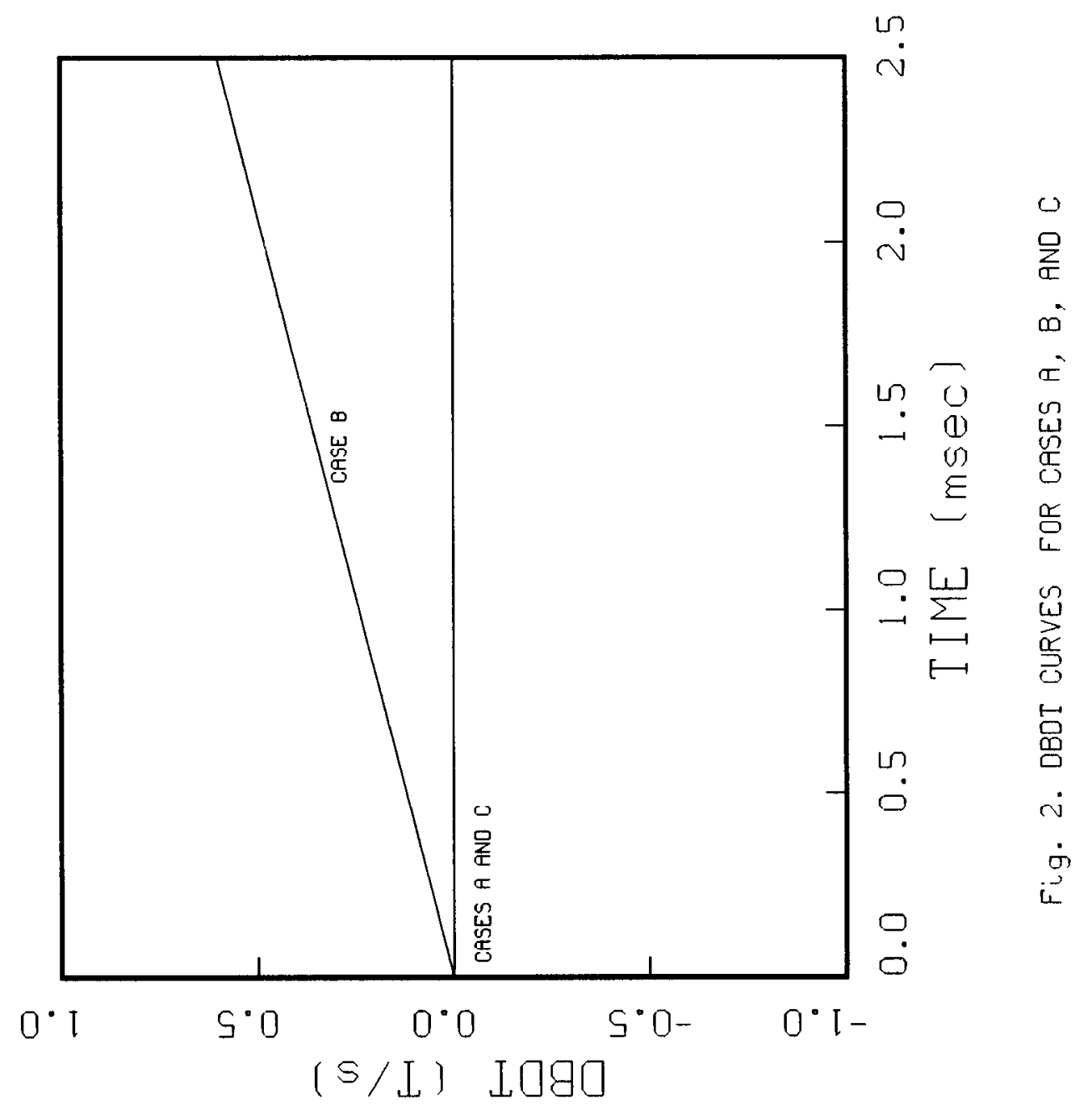




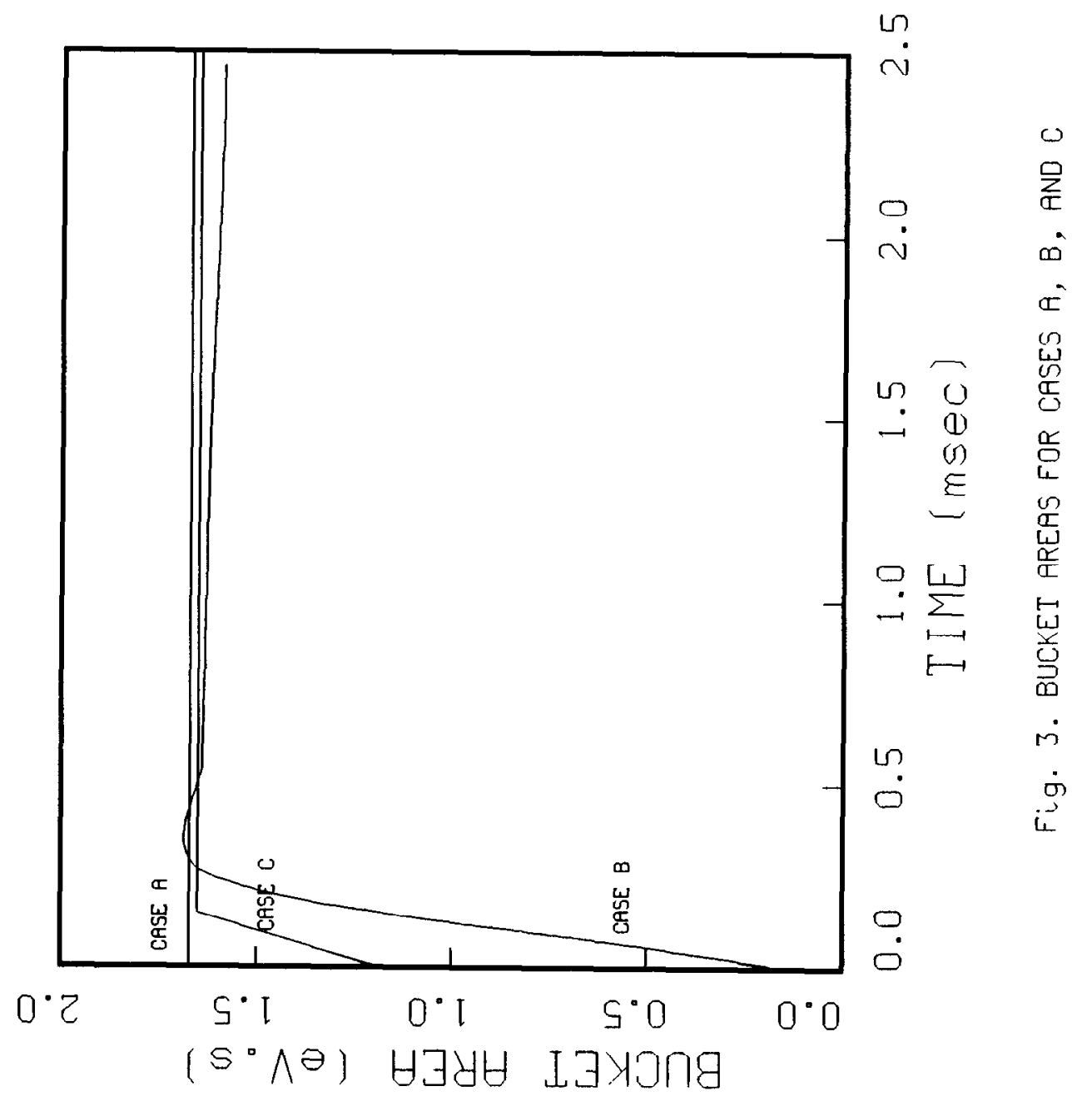




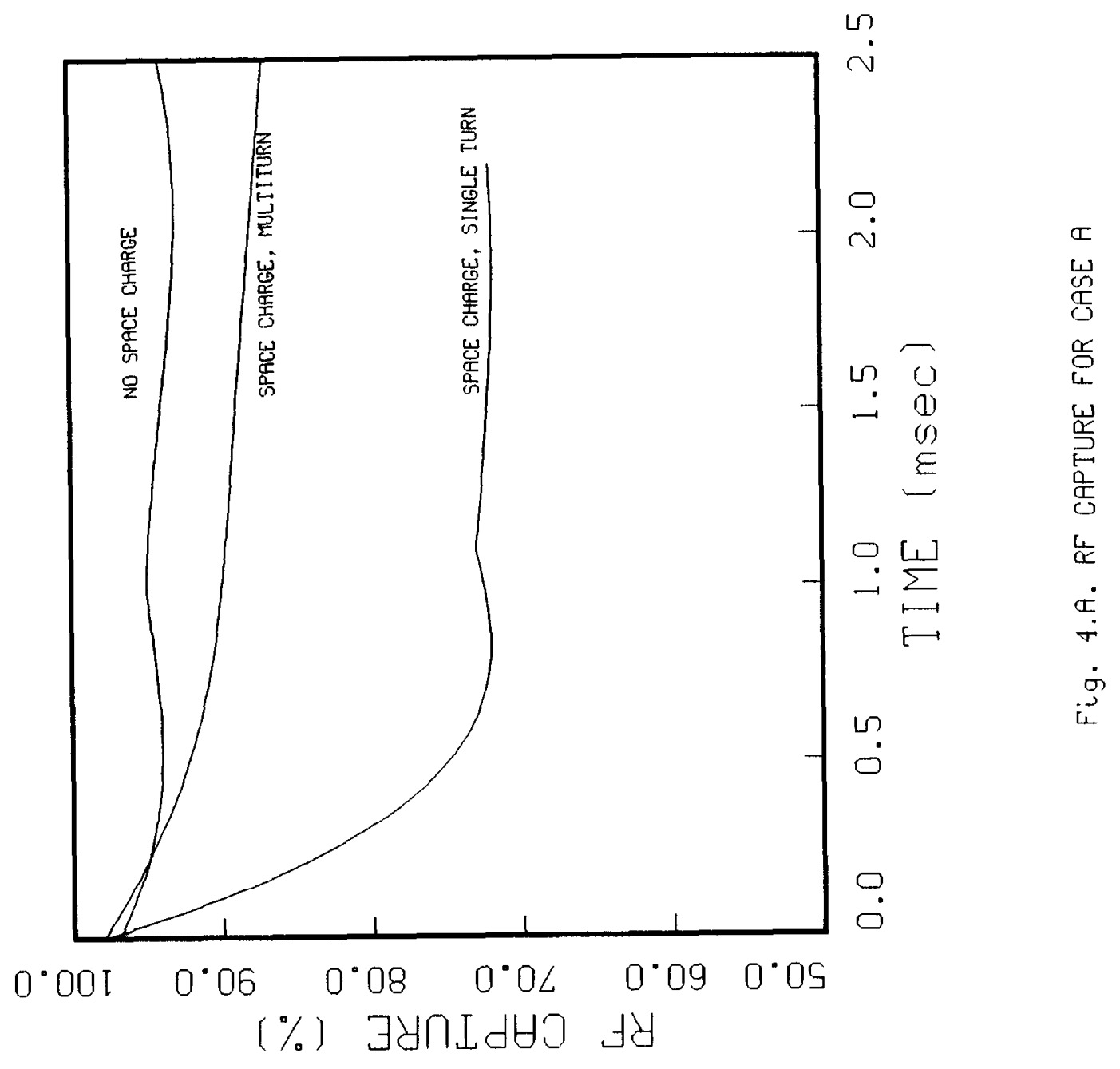




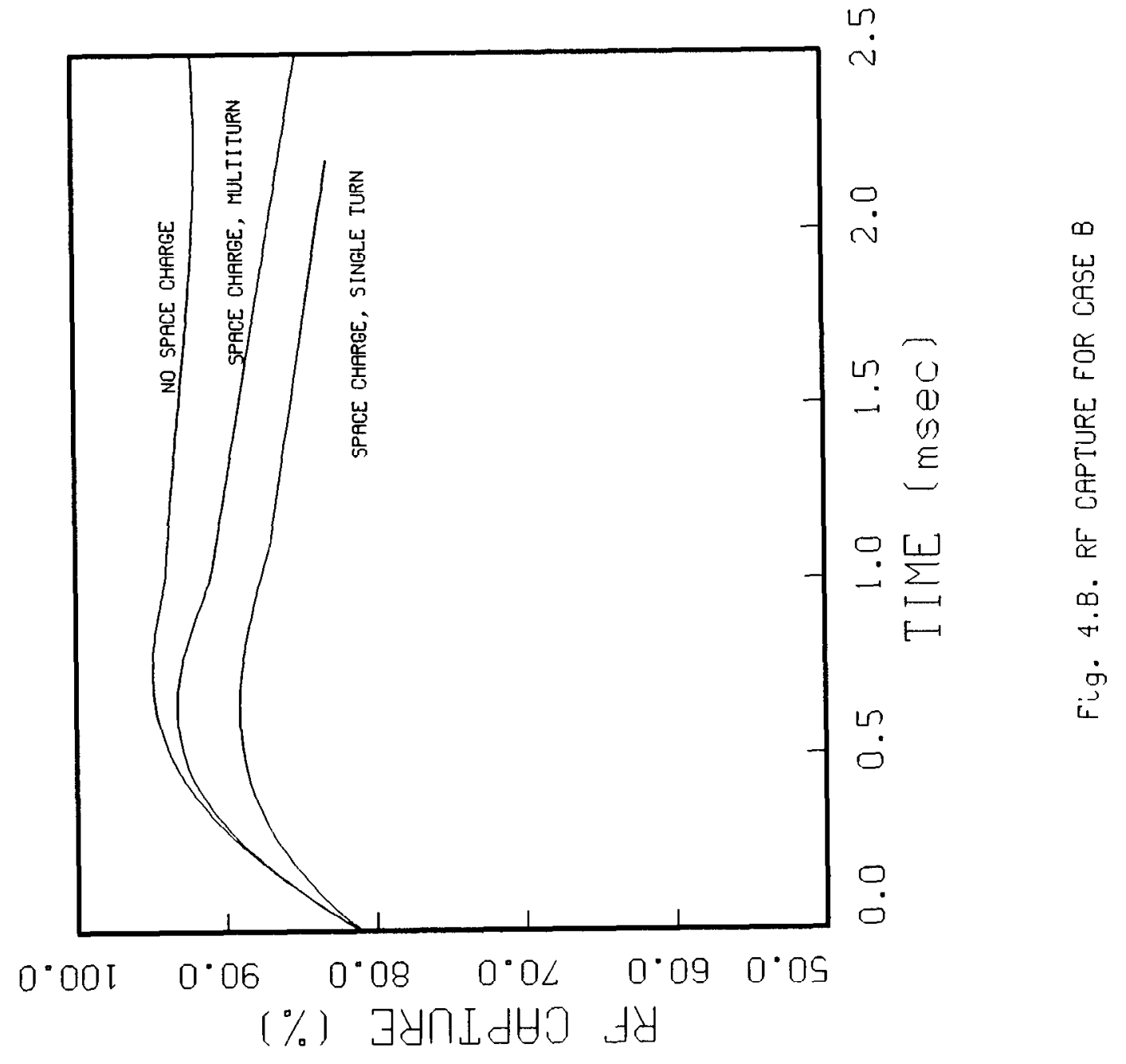




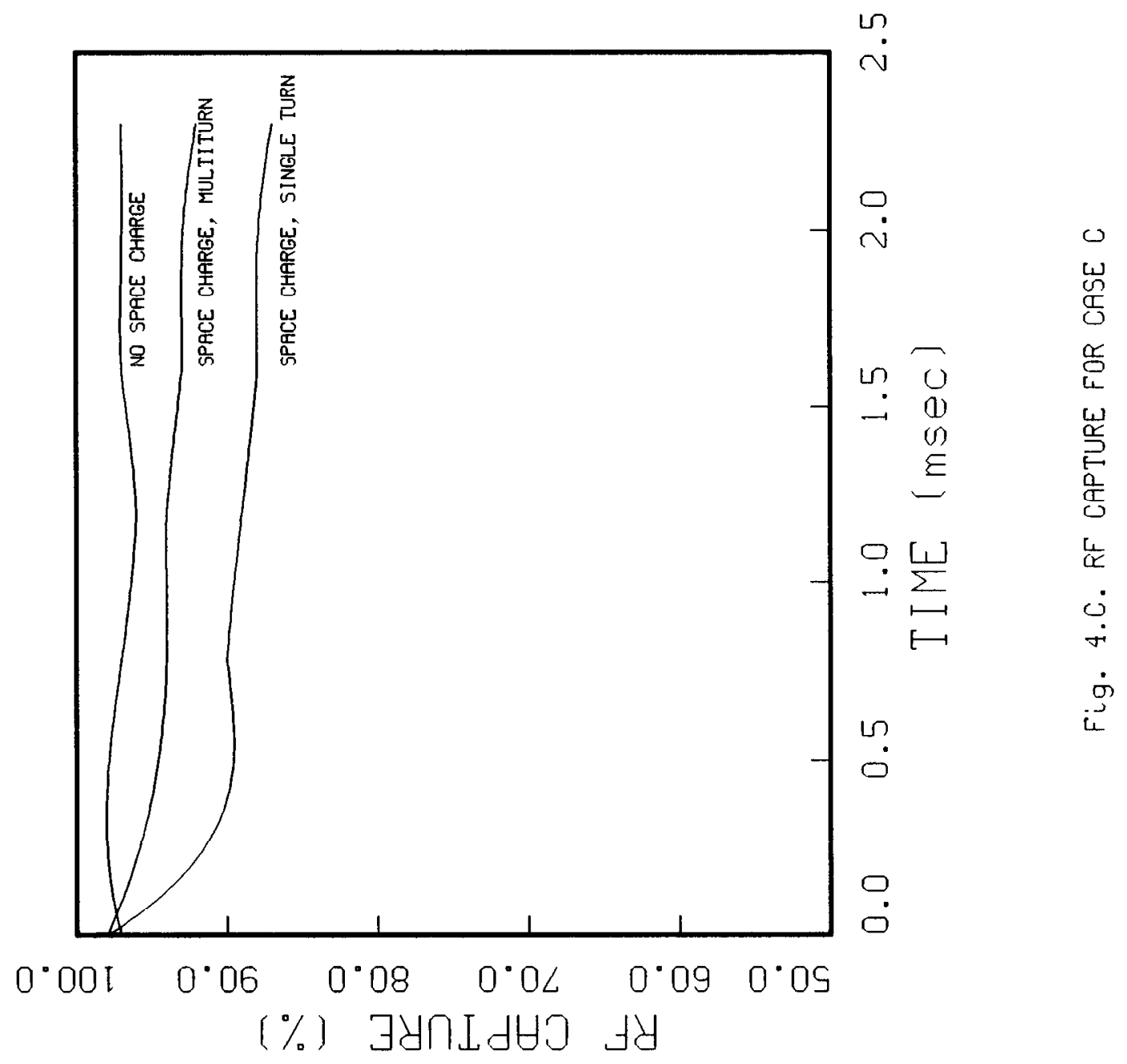




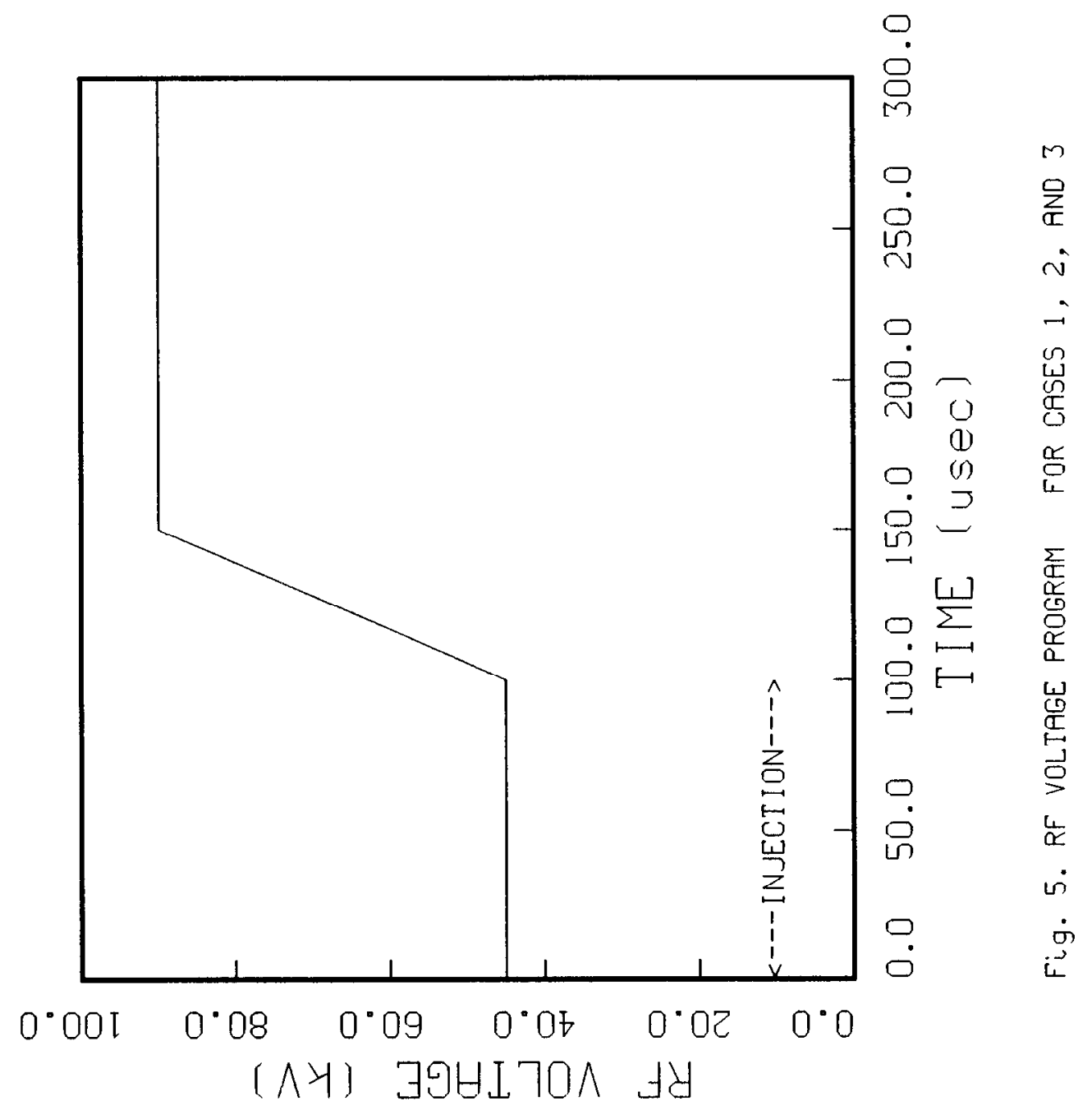




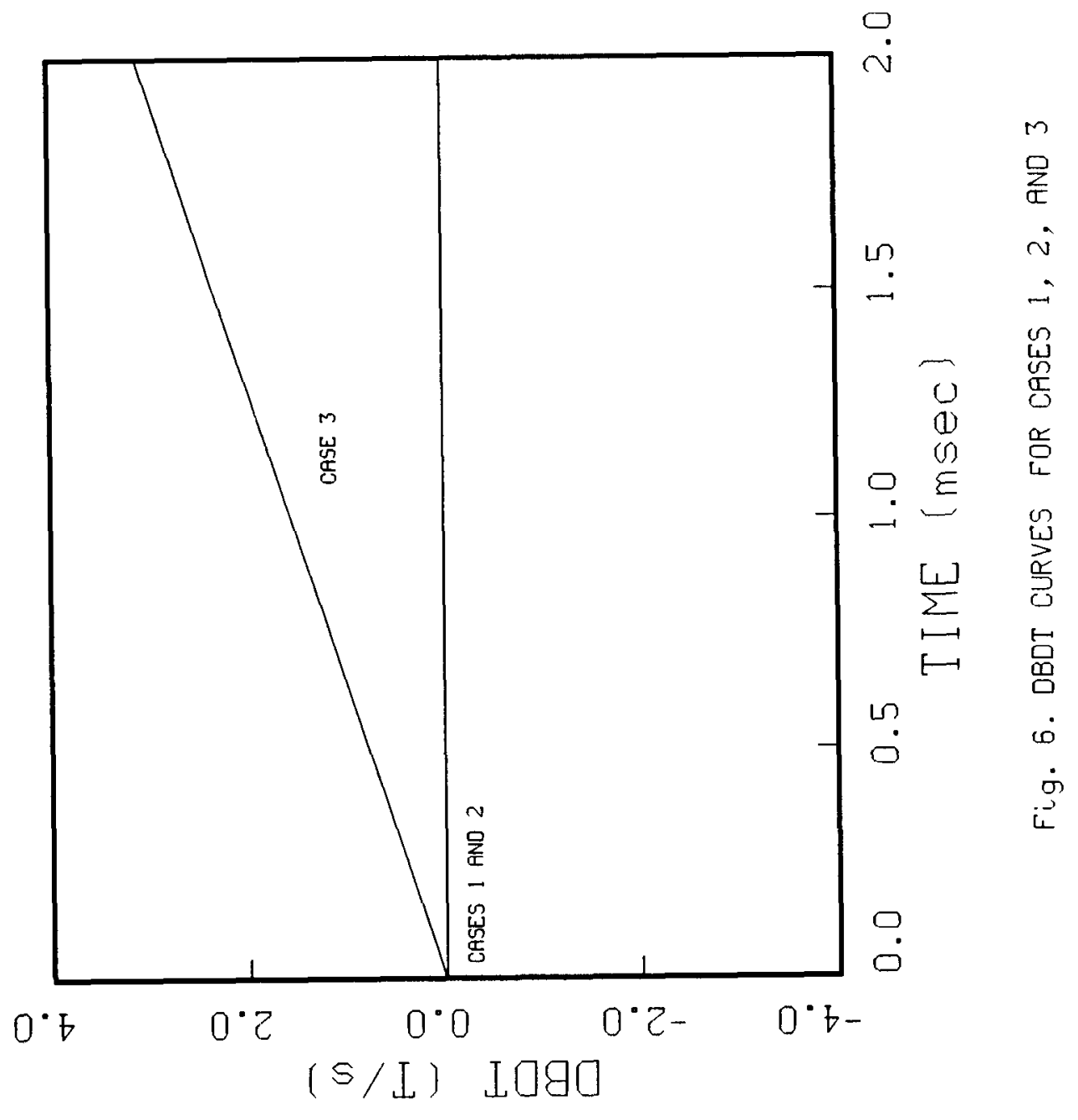




$\begin{array}{lllll}\text { Case \# } & \text { Space charge } & \dot{B} & \text { Bucket Area } & \text { Capture } \\ & {[\text { protons/bunch] }} & {[T / s]} & {[\mathrm{eV.s}]} & {[\%]} \\ 1 & 0 & 0 & 1.7 & 98 \\ 2 & 510^{12} & 0 & 1.7 & 95 \\ 3 & 510^{12} & \text { Linear } & 1.4 & 87\end{array}$

Table 3 Listing of Capture and Bucket Area at $2 \mathrm{~ms}$ for Cases 1, 2, and 3 


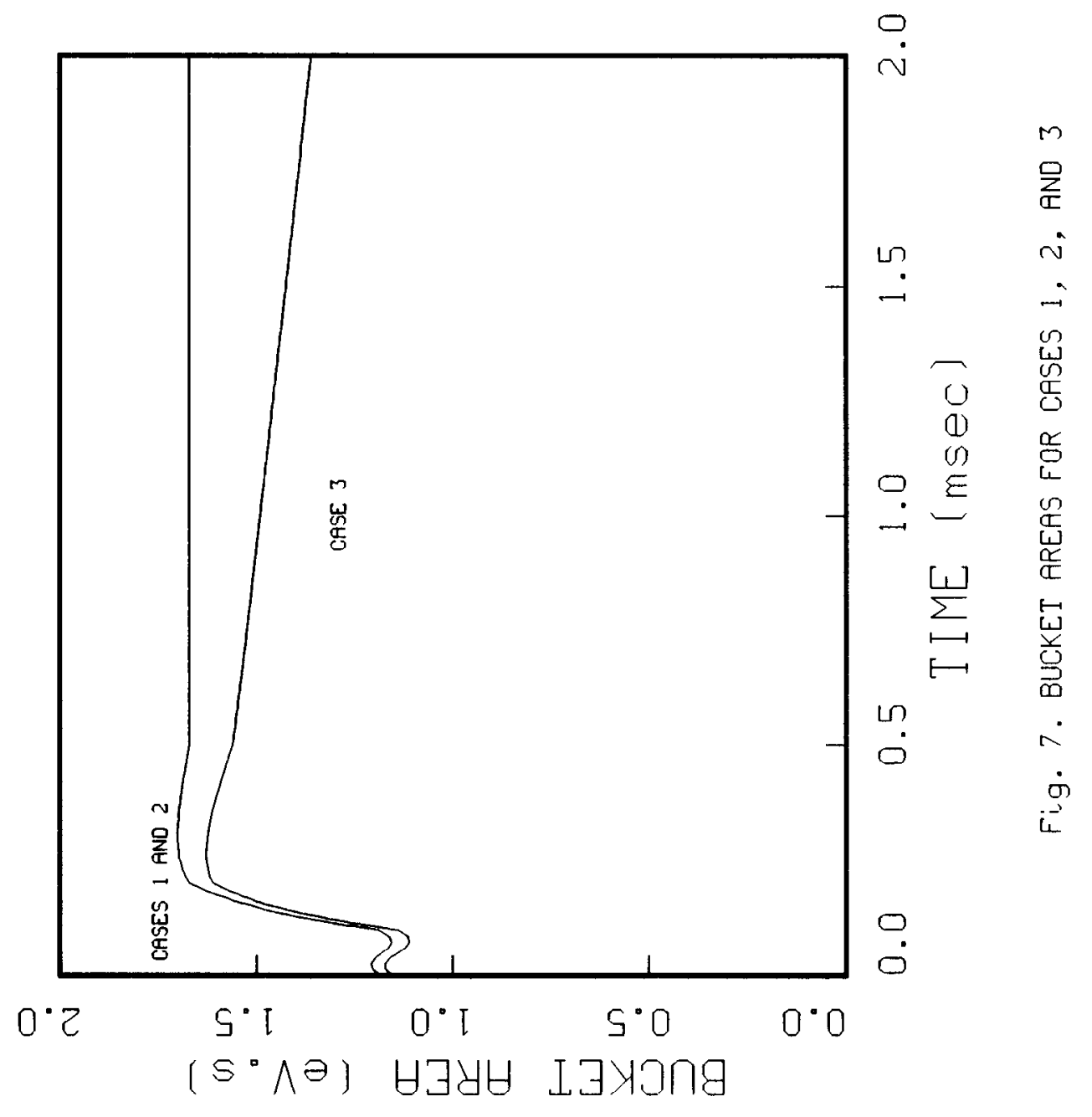




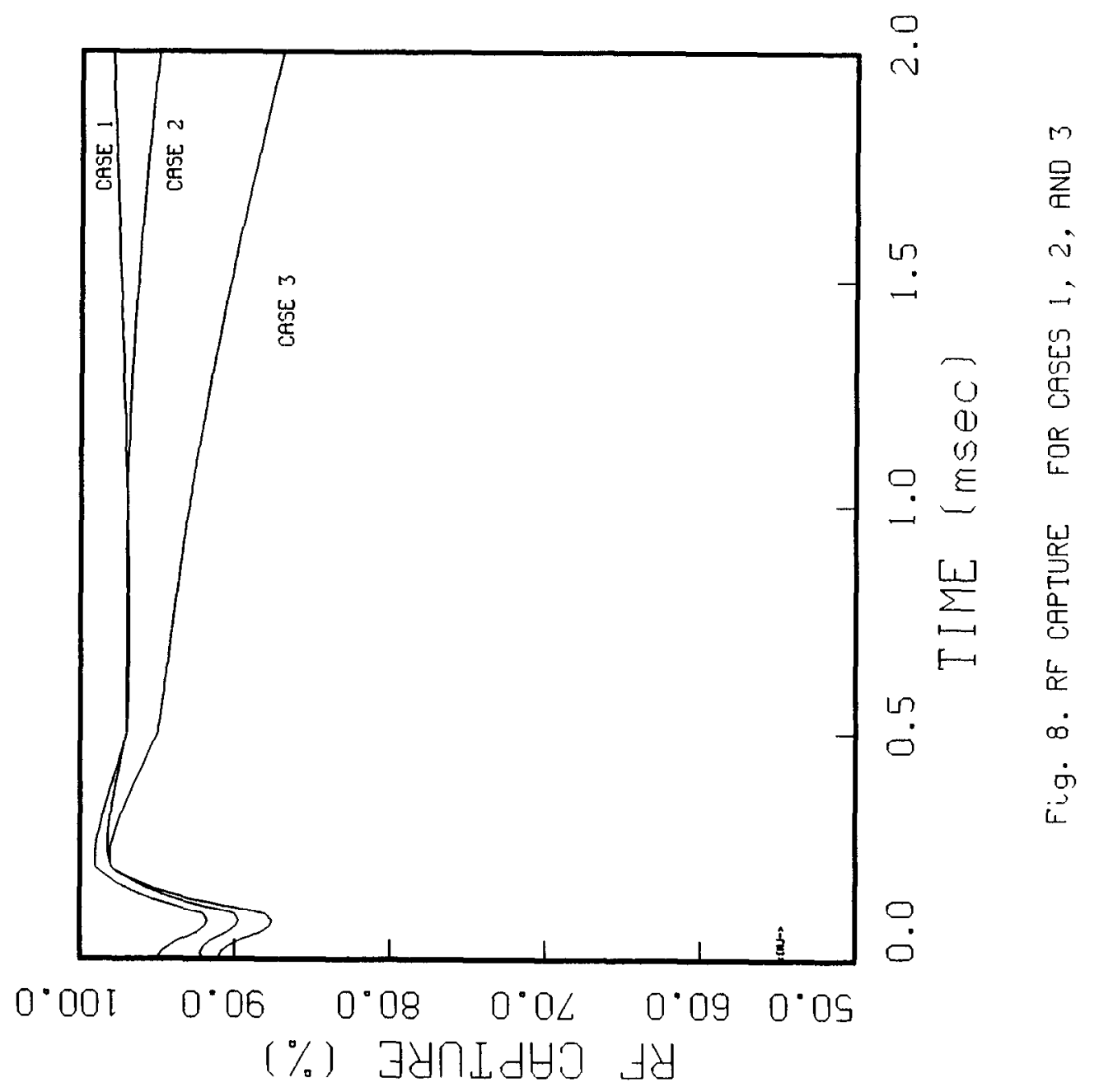




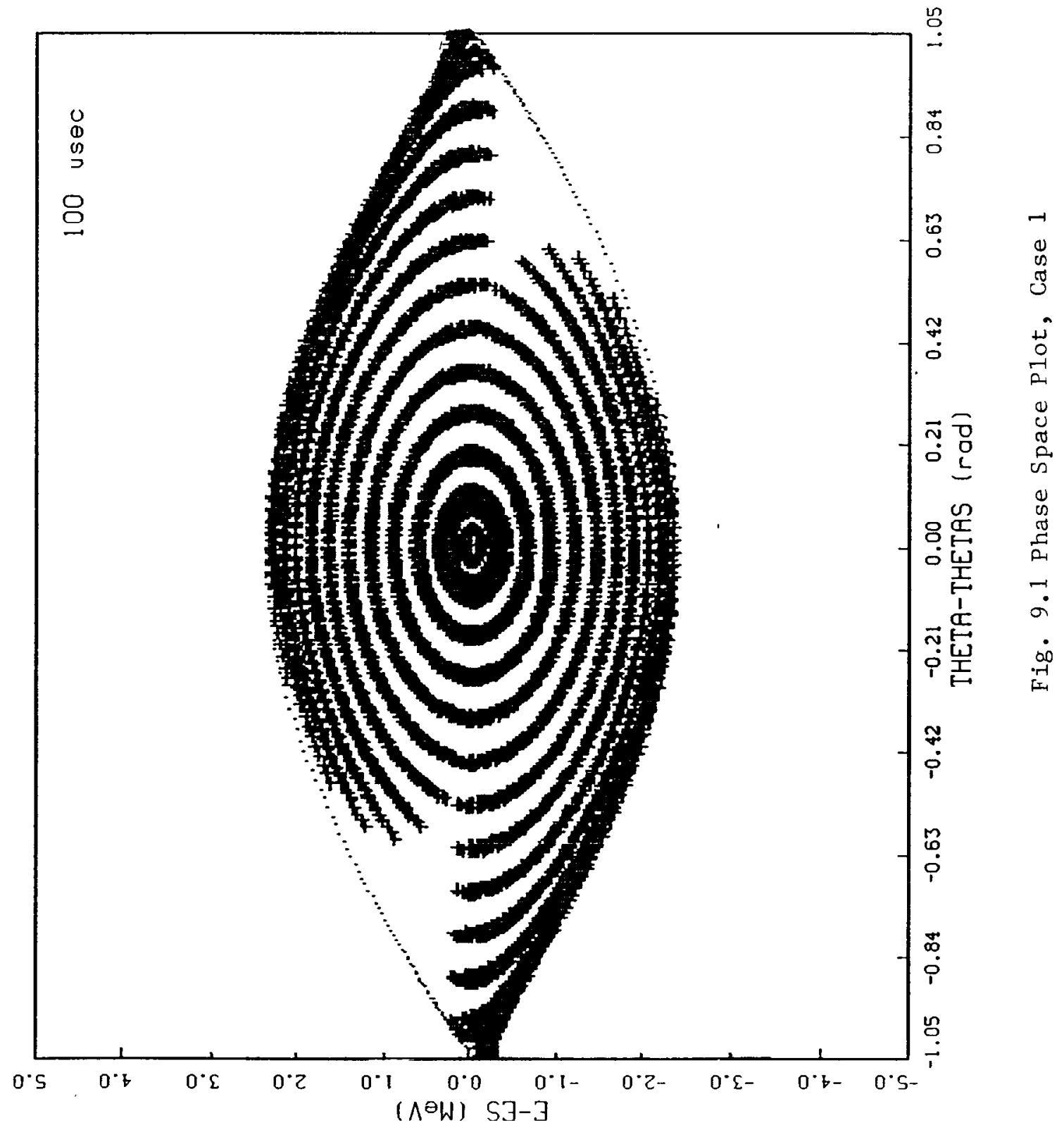




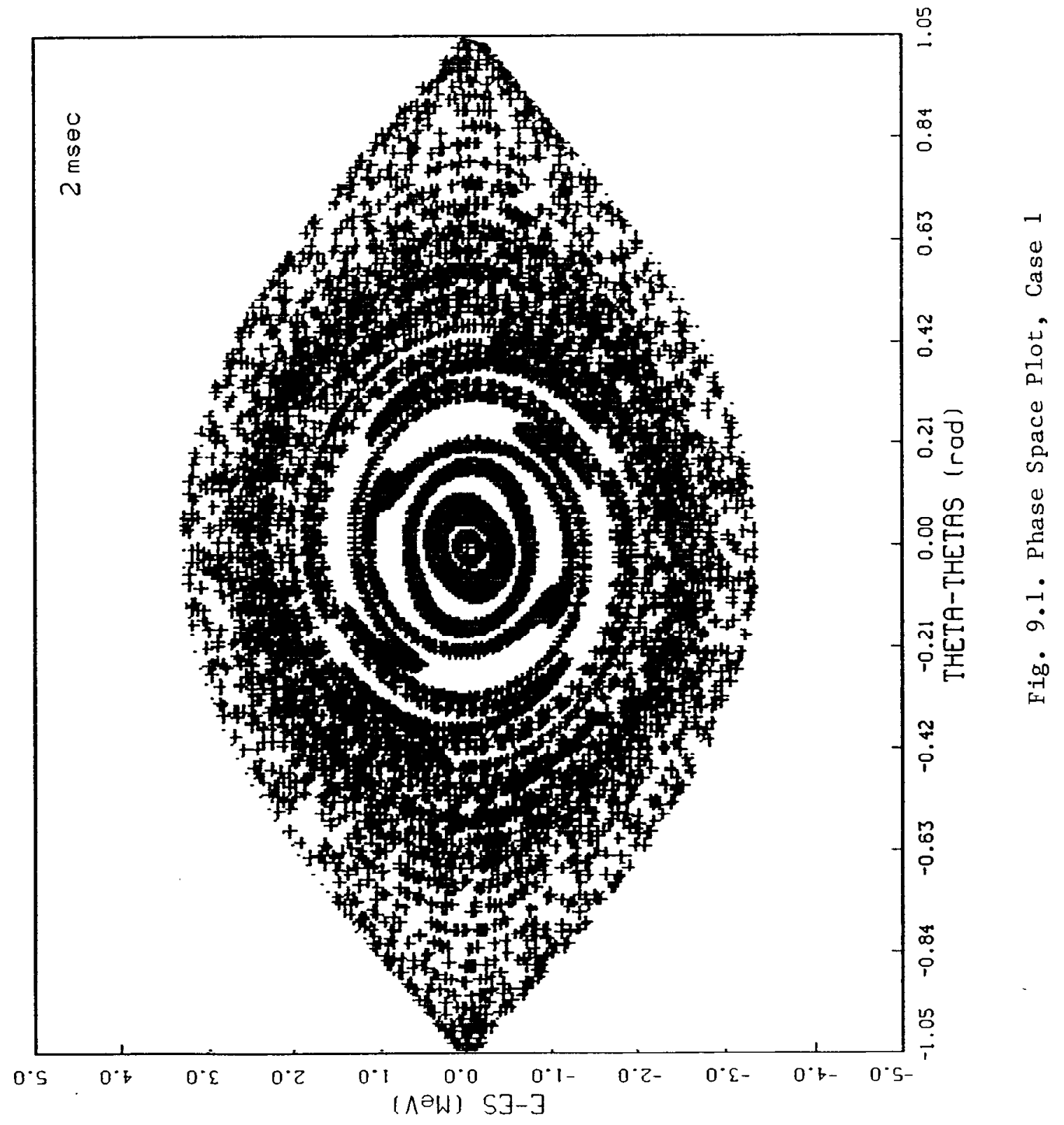




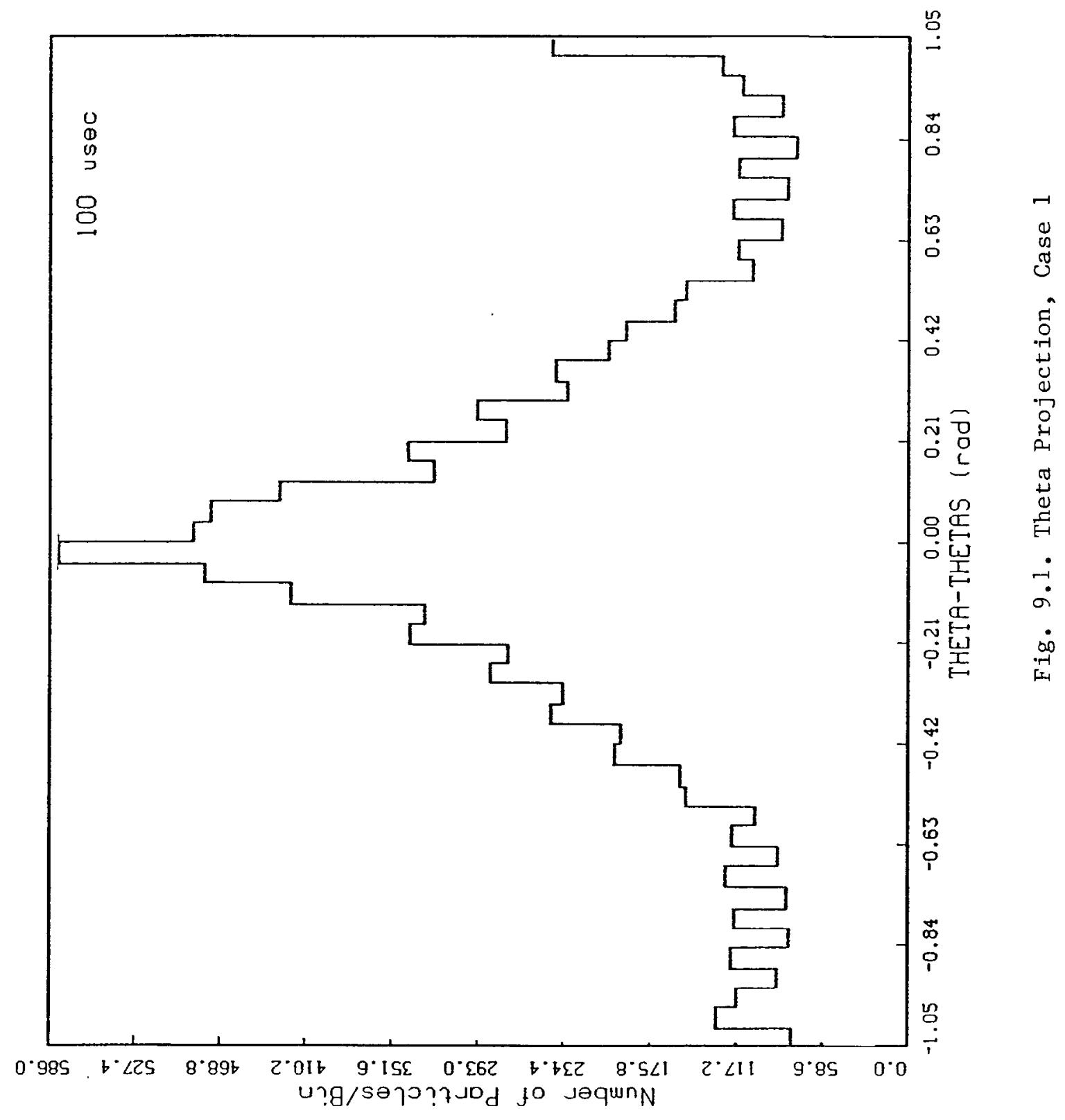




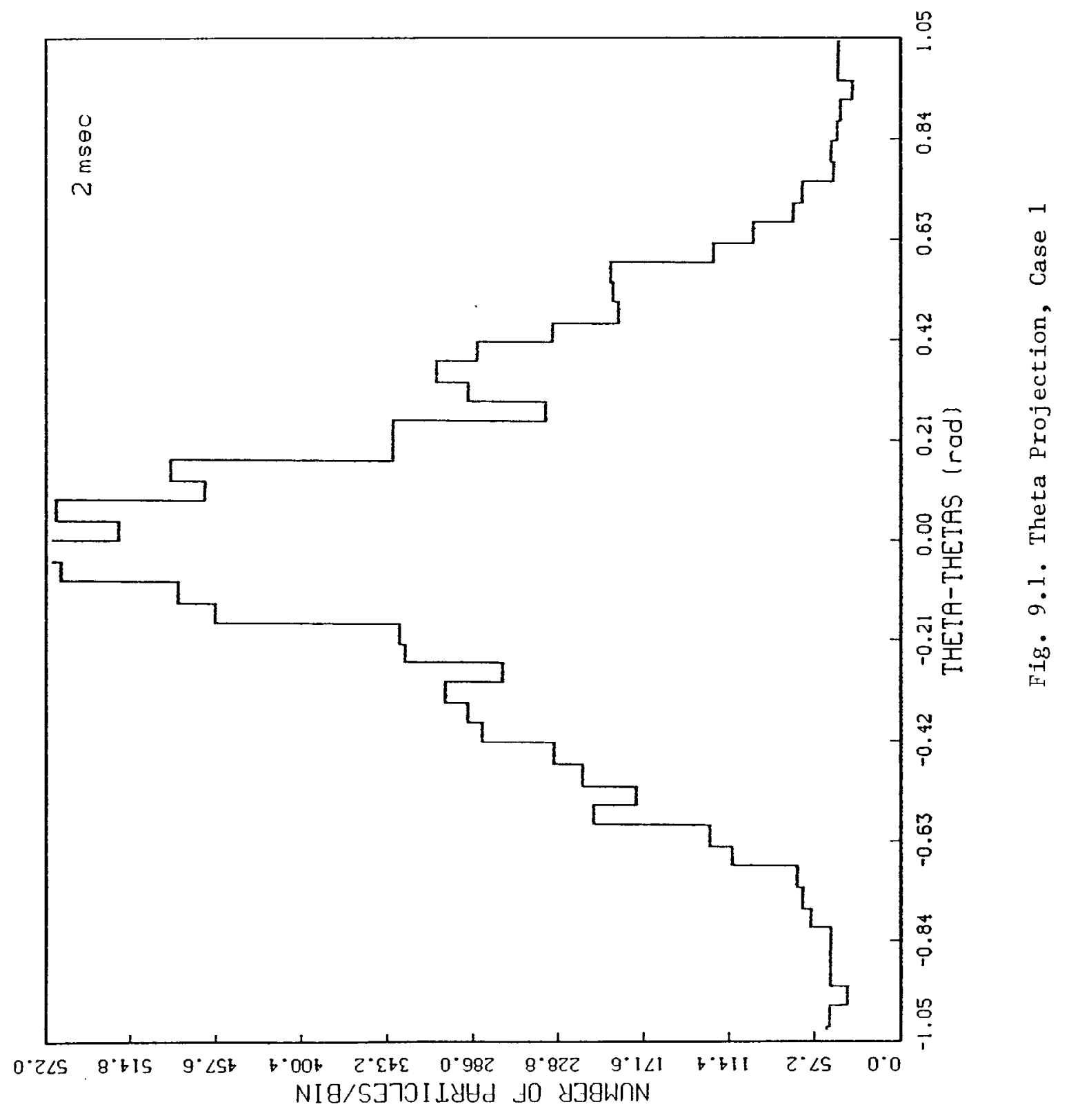




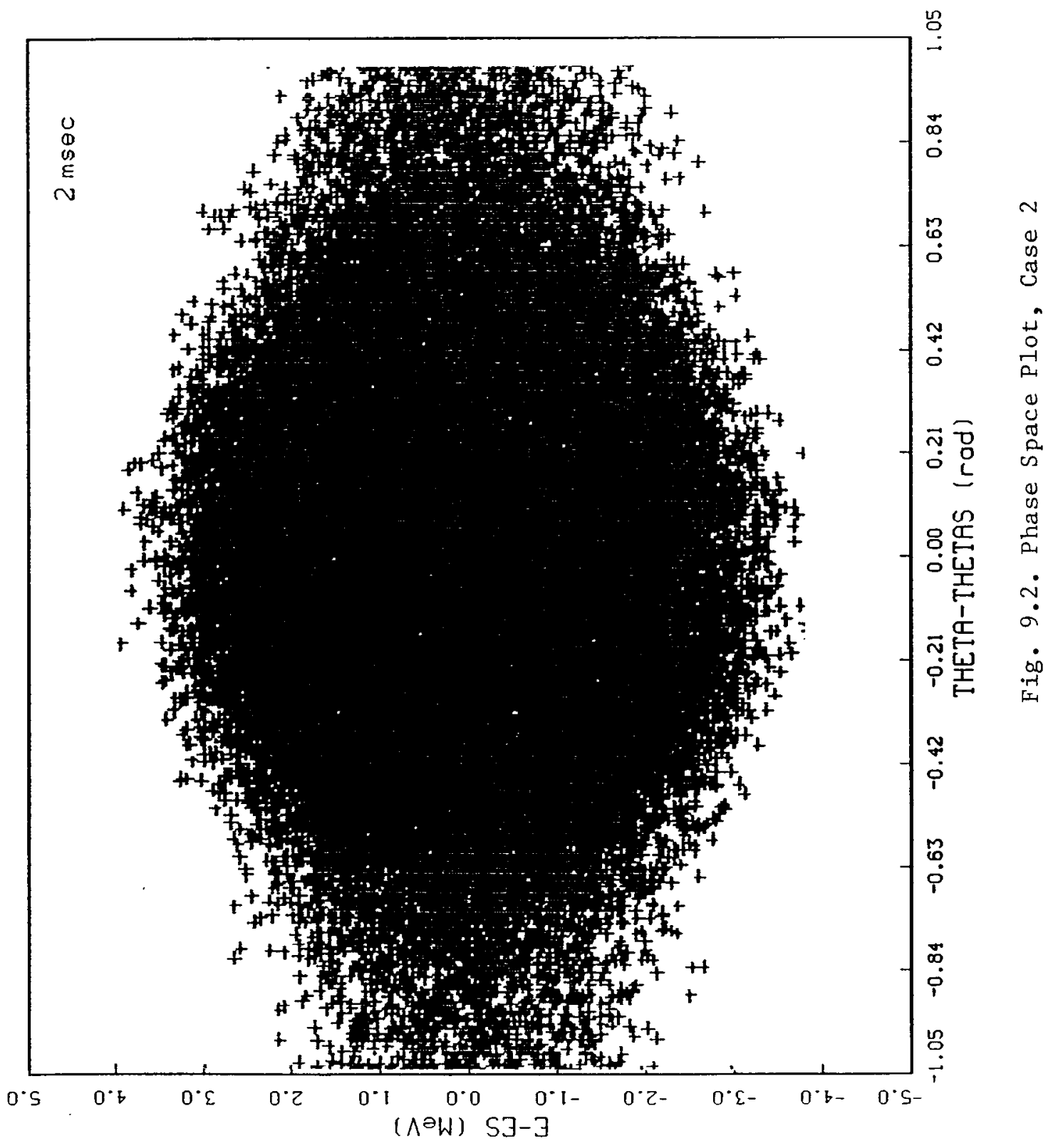




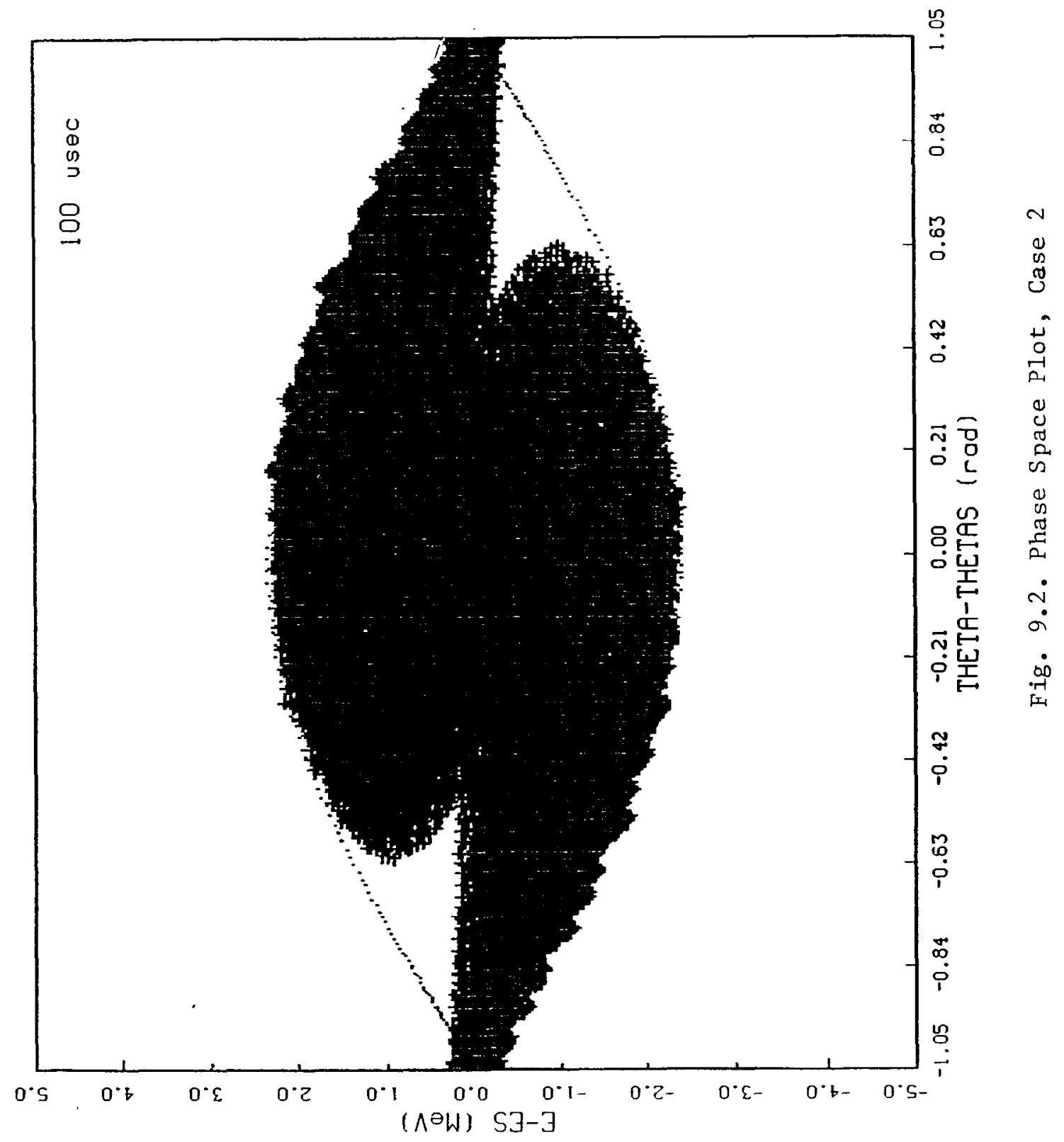




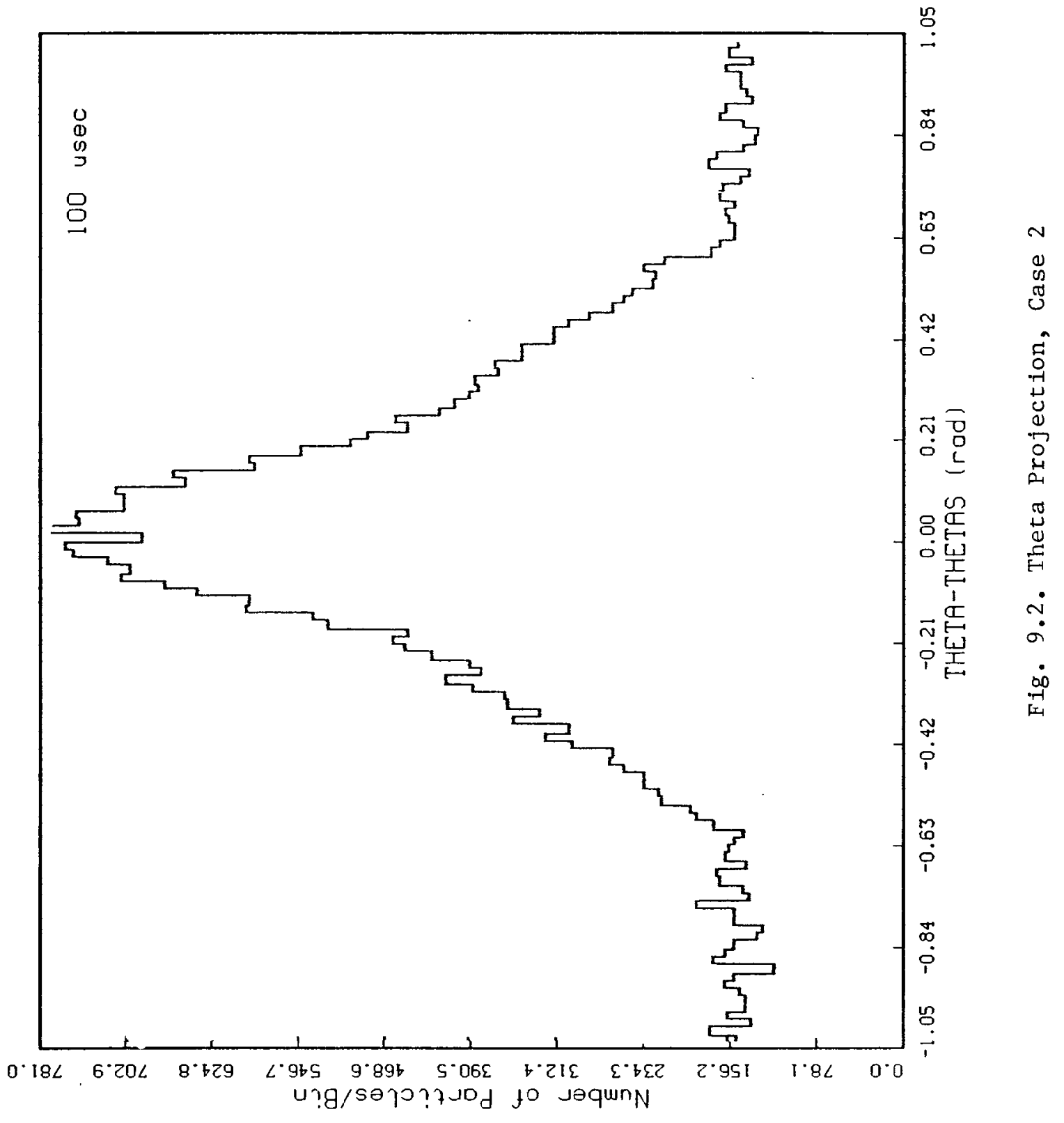




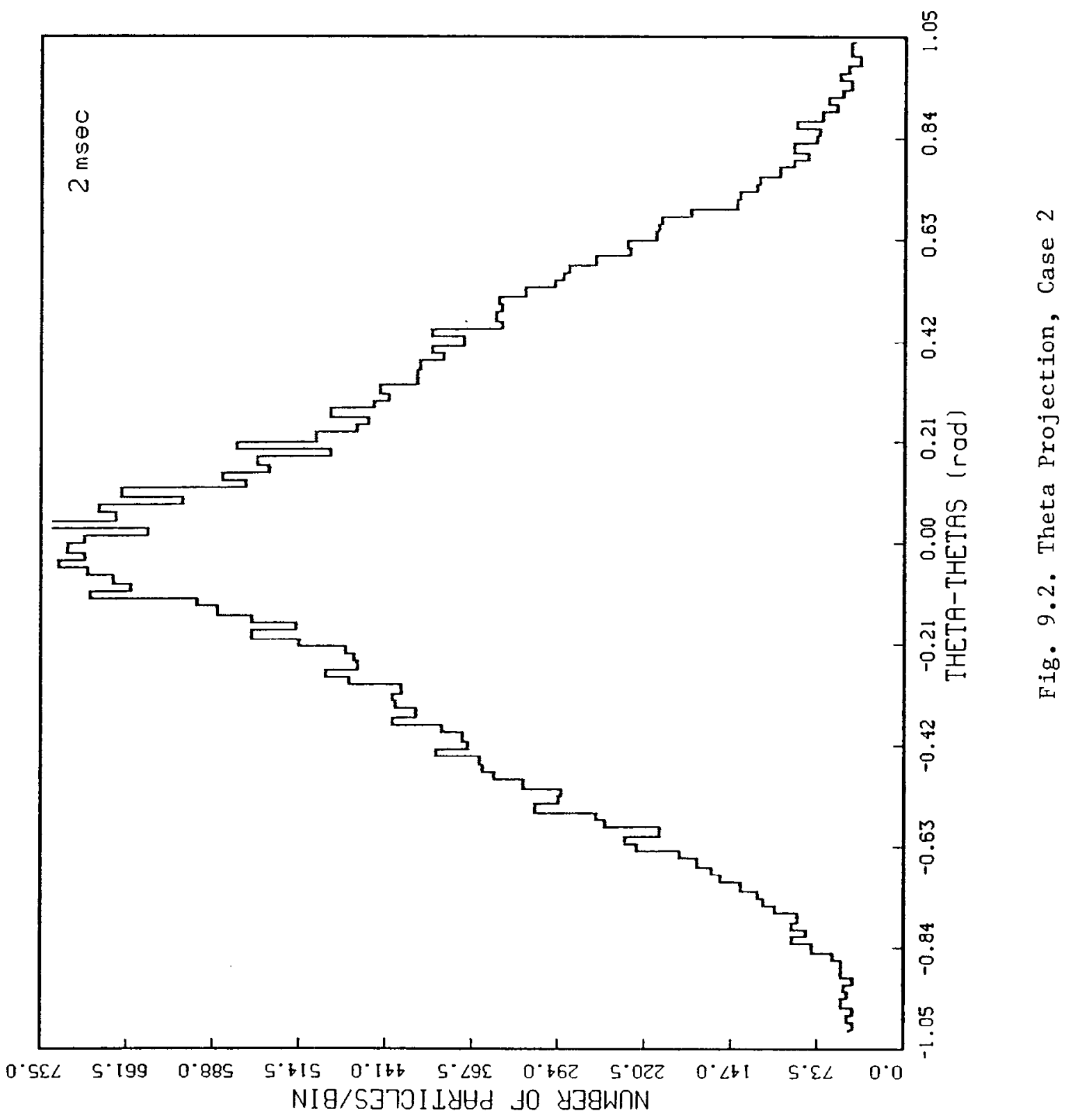




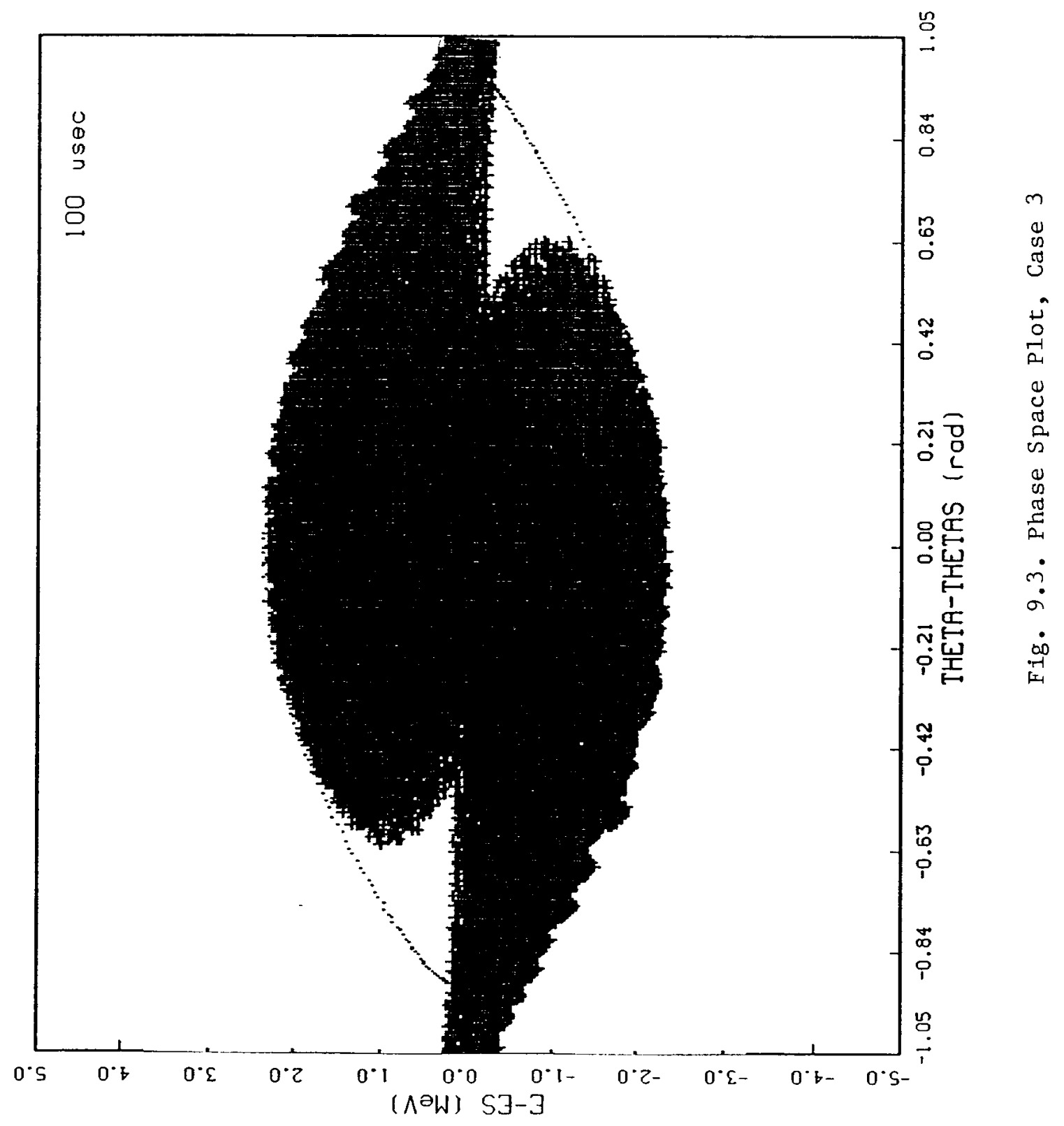




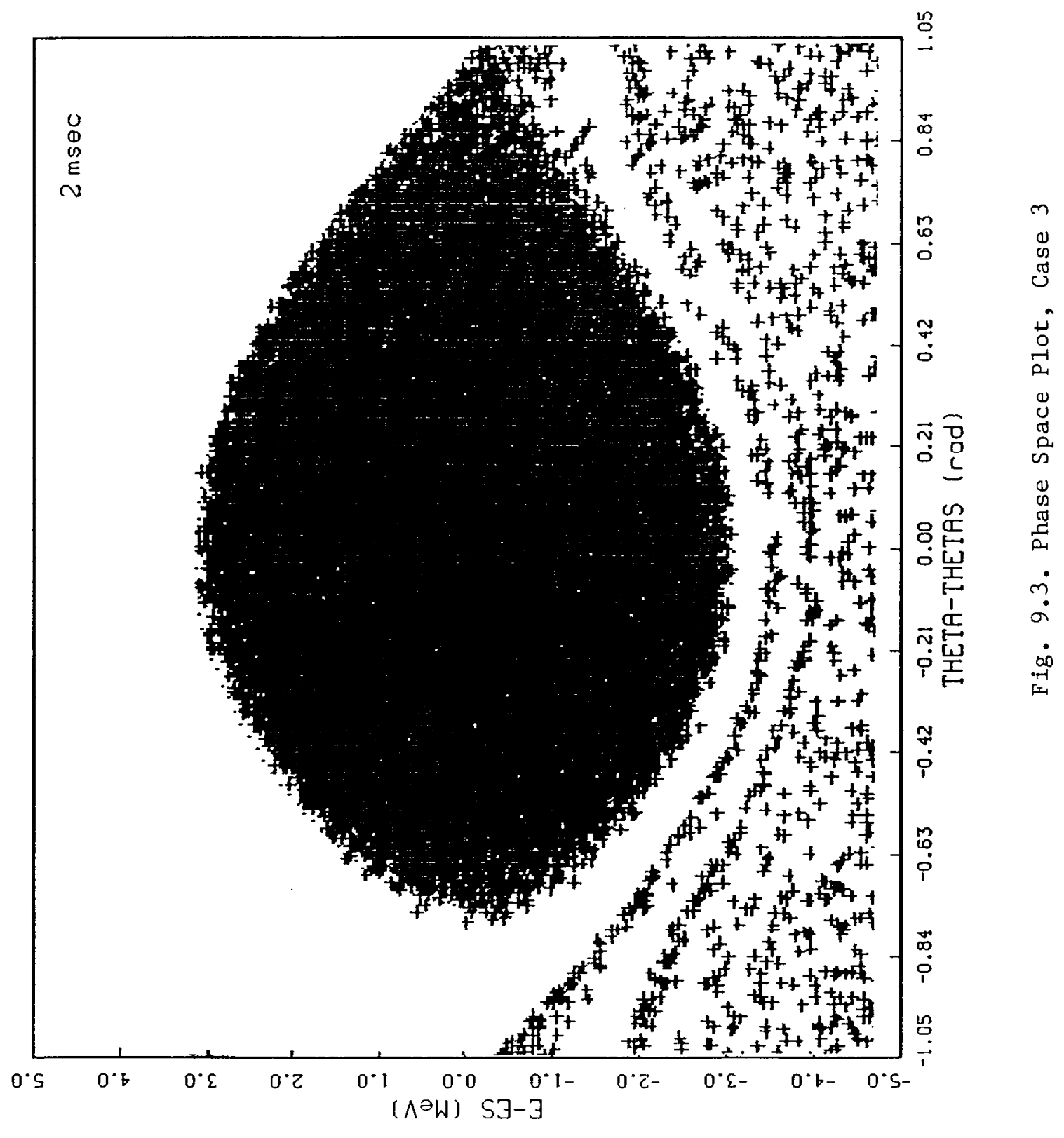




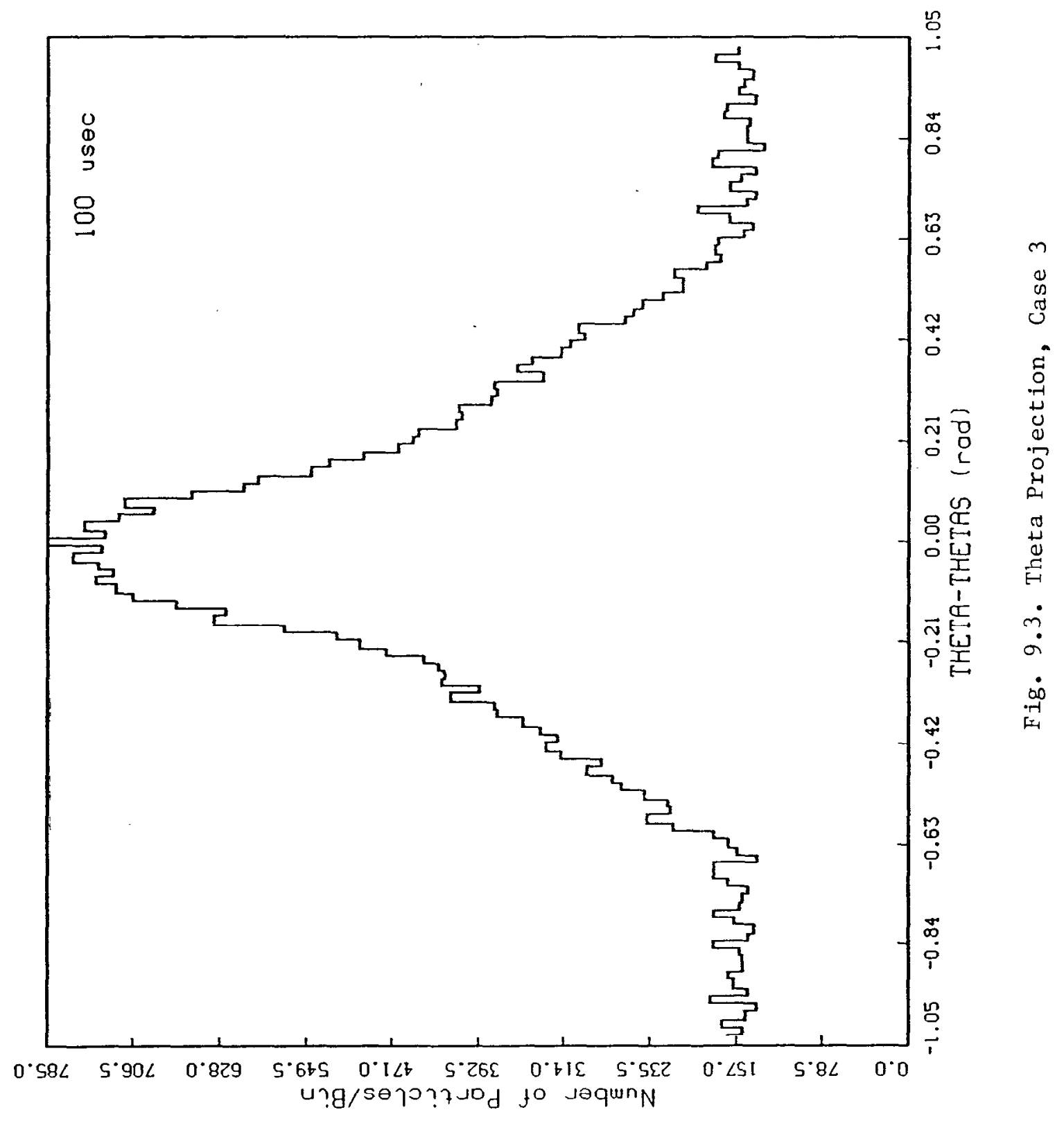




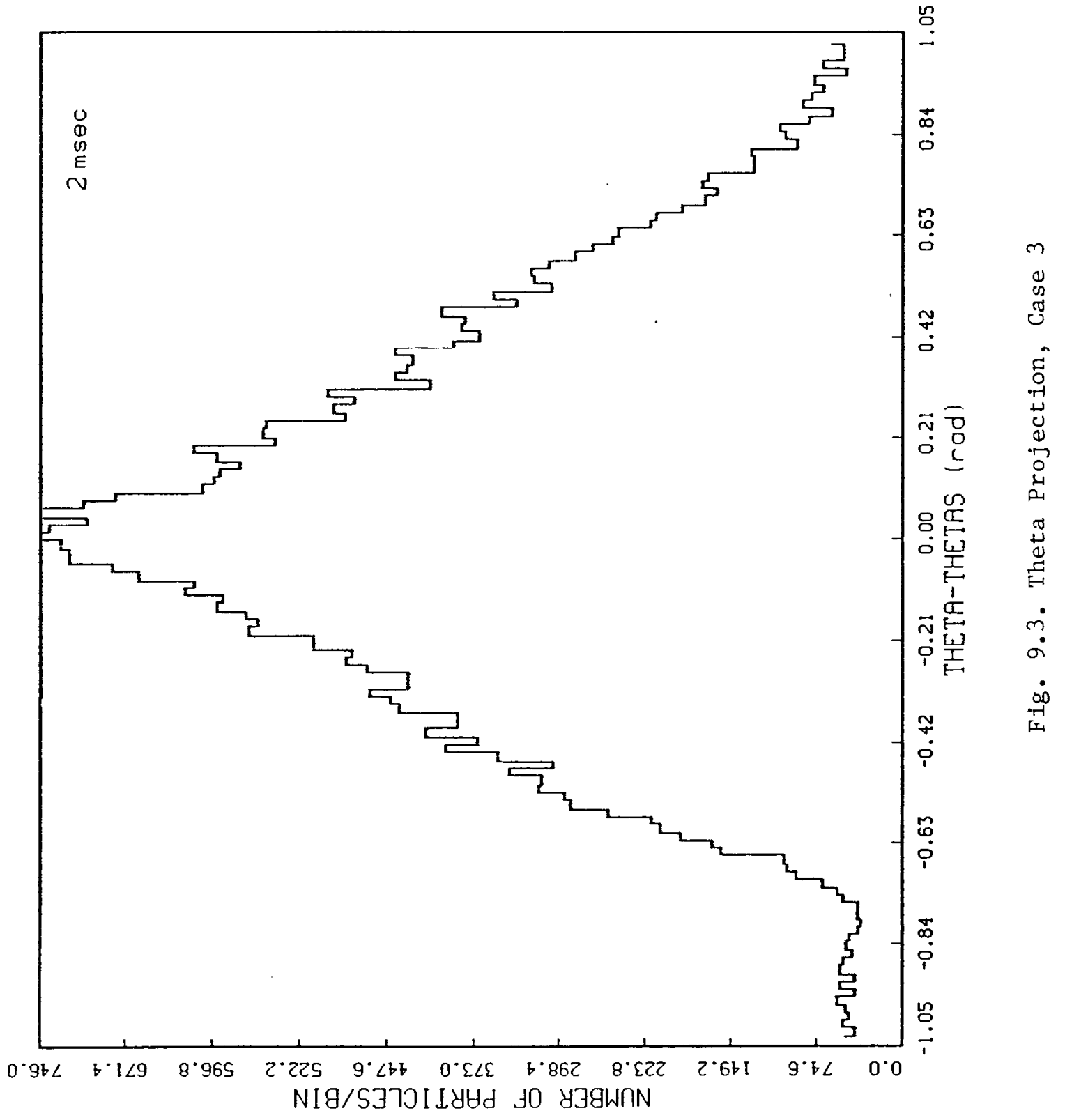

